\title{
Update in Antiepidermal Growth Factor Receptor Therapy in the Management of Metastatic Colorectal Cancer
}

\author{
Herbert H. Loong, ${ }^{1}$ Brigette B. Ma, ${ }^{1,2}$ and Anthony T. C. Chan ${ }^{1,2}$ \\ ${ }^{1}$ Department of Clinical Oncology, Prince of Wales Hospital, Shatin, New Territories, Hong Kong \\ ${ }^{2}$ State Key Laboratory in Oncology in South China, Sir YK Pao Centre for Cancer, Department of Clinical Oncology, \\ Hong Kong Cancer Institute and Li Ka Shing Institute of Health Sciences, The Chinese University of Hong Kong, \\ New Territories, Hong Kong \\ Correspondence should be addressed to Brigette B. Ma, brigette@clo.cuhk.edu.hk
}

Received 1 December 2008; Accepted 30 January 2009

Recommended by Daniel Chua

The approval of anti-epidermal growth factor receptor (EGFR) monoclonal antibodies in the treatment of metastatic colorectal cancer (CRC) has expanded the armamentarium against this disease. This paper will review the historical progress and recent clinical developments of anti-EGFR therapies in the treatment of metastatic CRC. Novel strategies of targeting the EGFR pathway to improve efficacy as well as ongoing research in identifying specific molecular predictors of response will be discussed.

Copyright () 2009 Herbert H. Loong et al. This is an open access article distributed under the Creative Commons Attribution License, which permits unrestricted use, distribution, and reproduction in any medium, provided the original work is properly cited.

\section{Introduction}

A decade ago, the systemic treatment of colorectal cancer (CRC) consisted only of fluoropyrimidine-based chemotherapy administered alone or in combination with either oxaliplatin or irinotecan in an empirical fashion, guided by serial measurements of radiological response. Owing to the remarkable advances in our understanding of the molecular mechanisms of carcinogenesis, target-based therapies are now commonly used as in the treatment of many types of cancer, including CRC. Cetuximab and panitumumab are monoclonal antibody against the epidermal growth factor receptor (EGFR) that has been approved for the treatment of patients with metastatic CRC $[1,2]$. The optimal clinical application of anti-EGFR agents in the management of CRC patients and the identification of predictive markers are the main focus of research in recent years. This article will concentrate on the developments and controversies of antiEGFR therapy in the management of CRC.

\section{EGFR as a Target in Colorectal Cancer}

The concept of manipulation of EGFR in the treatment of epithelial malignancies such as colorectal and lung cancers has actually been envisaged since the mid 1960s [1, 2]. It was during that period that the EGFR protein was first isolated, characterized, and recognized as a potential therapeutic target. Throughout the last 40 years, advances made in basic and clinical research have enhanced our understanding of this target, and many different classes of EGFR inhibitors are now at various stages of clinical development.

EGFR is a $170 \mathrm{kD}$ member of the ErbB receptor tyrosine kinase family of signaling proteins, and its ligands include epidermal growth factor (EGF), transforming growth factor$\alpha$ (TGF- $\alpha$ ), heparin-binding EGF (HB-EGF), and amphiregulin (AR). Ligand binding, dimerization, and phosphorylation of EGFR lead to activation of downstream proteins, leading to a cascade mediating cell growth and survival [3]. Two different anti-EGFR strategies are currently available in the therapeutic armamentarium: (1) monoclonal antibodies that prevent EGFR ligand binding and (2) tyrosine-kinase inhibitors (TKIs) that block phosphorylation of the intracellular tyrosine kinase component of the EGFR. Both of these strategies dampen signal transduction through some of the downstream pathways such as RAS/RAF/mitogenactivated protein kinase (MAPK) and phosphoinositide 3kinase (PI3K)-AKT cascades, thus limiting cell growth, proliferation, invasion, angiogenesis, and metastasis $[3,4]$. 
TABle 1: Comparison between cetuximab and panitumumab.

\begin{tabular}{lcc}
\hline & Cetuximab & Panitumumab \\
\hline Structure & Chimeric IgG-1, & Fully humanized: IgG-2 \\
& $30 \%$ murine & \\
Hypersensitivity reaction & $3 \%$ & $1 \%$ \\
Half life & 5 days & 7.5 days \\
Treatment schedule & $1-2$ weekly & 2-weekly \\
Antibody-dependent cell & Yes & \\
mediated cytotoxicity & Fc Domain & No ADCC reported \\
(ADCC) & of IgG-1 & \\
\hline
\end{tabular}

\section{Anti-EGFR Monoclonal Antibodies}

Cetuximab (chimeric IgG1 monoclonal antibody) and panitumumab (fully humanized IgG2 monoclonal antibody) are two anti-EGFR monoclonal antibodies currently approved in the treatment of metastatic CRC. The structural difference between the IgG-1-based cetuximab and IgG-2-based panitumumab is believed to have implications on their mechanisms of action. Preclinical studies have suggested that the cetuximab molecule is able to induce antibodydependent cell-mediated cytotoxicity (ADCC) $[4,5]$, where natural killer cells, monocytes, and eosinophils are recruited to lyse the targeted cells (i.e., tumor cells). Being a chimeric antibody, cetuximab is also associated with a slightly higher incidence of hypersensitivity and infusional reactions when compared with fully humanized panitumumab (see Table 1).

3.1. Cetuximab. Pharmacokinetics studies have shown that cetuximab's binding affinity for EGFR was shown to be one log higher than its natural ligand and its mechanism of action is thought to be competitive inhibition of ligand binding to EGFR. Cetuximab alone resulted in in vitro and in vivo growth inhibition in multiple tumor types, including CRCs $[4,5]$. Cetuximab was able to enhance the antitumor effect of irinotecan, as evident from an experiment on the HT-29 CRC xenograft model, where cetuximab and irinotecan given in combination resulted in a greater degree of tumor growth delay than when either agent was given alone [5]. Furthermore, cetuximab has been shown to overcome acquired resistance against irinotecan in vivo. This was shown as an experiment where the addition of cetuximab resulted in shrinkage of tumor xenografts which were otherwise progressing after previous treatment with irinotecan.

The landmark BOND trial [2] randomized 329 patients with metastatic CRC which were EGFR-positive and refractory to irinotecan, to either cetuximab alone or in combination with irinotecan in a $2: 1$ ratio. EGFR positivity was defined as $1+$ staining by immunohistochemistry, and "irinotecan-refractory" status was defined as disease progression on or within 3 months of irinotecan-based therapy. Cross-over was allowed from the monotherapy arm to the combination arm upon disease progression. Objective response rate (ORR) was significantly in favor of the combination arm $(22.9 \%$ versus $10.8 \%, P=.007)$. Fifty-six patients who were randomized to cetuximab alone eventually crossed over to the combination arm, while $3.6 \%$ and $35.7 \%$ of these patients achieved partial response and stable disease, respectively. This study led to the US Food Drug Administration (FDA) approval of cetuximab in patients with irinotecan-refractory meta static CRC. Subsequently, the NCIC-CO.17 study randomized patients who had failed at least 2 lines of prior therapies, to either supportive care or cetuximab alone [6]. In this study where no cross-over was allowed, there was a statistically significant advantage in median overall survival (OS) favoring the cetuximab arm (6.1 months) compared with supportive care (4.6 months). Partial responses occurred in 23 patients (8.0\%) in the cetuximab group but none in the group assigned to supportive care alone $(P<.001)$.

Cetuximab has also been investigated in the first-line setting. The "CRYSTAL" [7] study is a multicentre phase III trial which randomized more than 1000 patients with metastatic CRC, to either the "FOLFIRI" regimen alone (Irinotecan, infusional 5-fluorouracil and leucovorin in a 2 -weekly schedule), or in combination with cetuximab at a weekly schedule. The primary endpoint (progression-free survival (PFS)) was met in the study, where patients randomized to the combination arm had a significantly longer progression-free survival (8.9 months versus 8.0 months; $P$ $=.036)$ than the chemotherapy alone arm, but there was no difference in overall survival in the initial intention-to-treat analysis. Response rate was also significantly better in the combination arm $(46.9 \%$ versus $38.7 \%$; $P=.005)$, resulting in a larger number of patients being down staged enough to undergo resection of liver metastases (9.8\% versus $4.5 \%)$. The "OPUS" study [8] is another first-line randomized phase II study, which randomized 337 chemotherapy-nave patients with metastatic CRC, to either the FOLFOX-4 regimen or in combination with cetuximab in a 1:1 fashion. The overall RR was $45.6 \%$ in the combination arm versus $35.7 \%$ in FOLFOX-4 alone arm. In the "ACROBAT" study, Tabernero et al. reported on 42 patients who were treated with FOLFOX-4 plus cetuximab, showing a confirmed ORR of $81 \%$ [9]. Encouragingly, 10 patients (23\%) underwent resection of previously unresectable metastases, 8 of them had liver metastases. The resection with curative intent rate of $23 \%$ achieved in this study is therefore comparable with the highest reported for unselected patients.

\subsection{Panitumumab. The US FDA approval of panitumumab was based on a pivotal multinational phase III study that involved over 400 patients [10]. This study compared panitumumab versus best supportive care (BSC), allowing cross-over from the BSC arm to panitumumab upon dis- ease progression. The median PFS time was 8 weeks for panitumumab and 7.3 weeks for BSC. After a 12-month followup period, response rates were $10 \%$ for panitumumab and $0 \%$ for BSC $(P<.0001)$. The lack of difference in OS (hazard ratio HR 1.00; 95\% confidence interval, CI 0.82 to 1.22) maybe attributed to the cross-over design, where $76 \%$ of patients in the BSC arm subsequently received panitu- mumab. As expected with anti-EGFR therapies, skin-related toxicities occurred in $90 \%$ of patients in the panitumumab group but no patients had grade 3 or 4 infusional reactions.}




\section{EGFR Tyrosine Kinase Inhibitors}

Although not approved for the treatment of CRC, small molecule inhibitors of the EGFR tyrosine kinase (TKI) have been shown to have meaningful activity in different tumor types such as lung and pancreatic cancer. In contrast to EGFR monoclonal antibodies, the site of action of these drugs is intracellular at the ATP-binding site of the EGFR TK domain. Compared with monoclonal antibodies, TKIs may potentially inhibit multiple targets and tend not to induce receptor downregulation.

4.1. Gefitinib. Gefitinib is a low-molecular-weight competitive inhibitor of ATP-binding pocket of the EGFR TK domain [11], which is approved for the treatment of nonsmall cell lung cancer. Clinical trials of gefitinib as a single agent in CRC reported no objective tumor responses [12-14] however, a sizable proportion of patients did have disease stabilization. A phase II trial which compared the $250 \mathrm{mg}$ versus a $500 \mathrm{mg}$ daily dosing of gefitinib [15], reported 1 partial response in a patient who received the $500 \mathrm{mg}$ dose. Paired biopsies pre- and posttreatment biopsies performed in 28 patients showed that $84 \%$ had no change or increase in the expression level of phosphorylated EGFR, MAP kinase, and Ki67 after treatment. Gefitinib and chemotherapy in combination have also been investigated in several trials, where the response rates seemed to be superior to the historically reported rates of chemotherapy alone [16-18]. However, there were significantly more toxicities particularly with respect to neutropenia and diarrhea with the combination. Studies combining gefitinib with irinotecan also resulted in greater toxicity, with some trials requiring early termination.

4.2. Erlotinib. Erlotinib has also been studied in advanced CRC. There have been mixed reports of some clinical activity when used as a single agent. As with gefitinib, erlotinib produced a higher response rate when combined with chemotherapy. High incidence of toxicity was noted when given in combination with systemic chemotherapy, especially in two trials where erlotinib was given in combination with FOLFOX and bevacizumab $[19,20]$, with the latter trial having prematurely closed due to toxicity.

\section{Incidence and Implications of Skin Rash}

Anti-EGFR monoclonal antibodies and EGFR TKIs are associated with a distinctive skin rash. The rash is characterized histologically as a neutrophilic infiltrate in perifollicular areas within the basal layer of the skin, which is different from that seen in typical acne. Skin toxicity is generally observed within 2 to 3 weeks after the start of treatment and gradually resolves in most patients, even when antiEGFR treatment is continued. In the BOND study, the most frequently observed adverse event to cetuximab was the skin rash, and in the panitumumab study, Hecht et al. [21] reported a 95\% incidence of acneiform skin rash of any grade. Grade 3 rash occurred in 3\% of patients and none experienced grade 4 skin toxicities. An association between the severity of acneiform rash and efficacy to cetuximab has been well described. Retrospective analysis of the BOND data showed a clear association between higher grades of skin reaction with RR and time to progression (TTP) [2]. This association is also seen with panitumumab [10] and seems to hold true in the treatment of tumors of other sites with this class of agents.

The "EVEREST" study [22] was a phase I/II dose-escalation study, where patients who were receiving cetuximab were randomized at 22 days, to either standard dose of weekly $(250 \mathrm{mg} / \mathrm{m} 2 /$ week $)$ cetuximab or an escalating dose of cetuximab until the development of grade 3 toxicity, with a maximum ceiling dose of $500 \mathrm{mg} / \mathrm{m} 2 /$ week. Skin and tumor biopsies were obtained. Preliminary report suggested that the PFS in standard-dose arm was 3.9 months and 4.8 months in the dose-escalated arm. Dose-related increases in pharmacokinetic parameters (e.g., Cmax, AUC) were observed in the escalated arm. The authors concluded that cetuximab dose escalation up to $500 \mathrm{mg} / \mathrm{m} 2 / \mathrm{w}$ may improve ORR in patients who experienced no or slight skin reactions on standard-dose cetuximab.

\section{Combining Targeted Therapies in Colorectal Cancer}

Combinations of multiple targeted agents with or without the additional of chemotherapy have also been investigated. The BOND-2 trial [23] randomized irinotecan-refractory patients to either 2 drugs (bevacizumab and cetuximab) or 3 drugs (bevacizumab, cetuximab and irinotecan). The results were encouraging, with the 3-drug arm resulting in a better TTP and ORR than the 2-drug arm. This study also showed for the first time that monoclonal antibodies in combination could induce an ORR of $20 \%$ in the absence of chemotherapy. Nonstatistical comparison with result of the BOND-1 study suggested that bevacizumab may enhance the effects of irinotecan-cetuximab combination, with an ORR of $37 \%$ as compared to $23 \%$ reported for the cetuximab/irinotecan arm $[2,23]$ (see Table 2). Subsequent to the BOND-2 study, 3 other randomized trials have been reported on the feasibility of combining monoclonal antibodies in the treatment of CRC. The PACCE study [24] randomized 824 treatment-nave patients with metastatic CRC to oxaliplatin-based chemotherapy plus bevacizumab, with or without panitumumab, and 230 patients to irinotecanbased chemotherapy plus bevacizumab with or without panitumumab. The study was terminated at a preplanned interim analysis after 231 events were reported in patients who received oxaliplatin-based therapy, where a statistically significant increase in PFS was reported in the arm without panitumumab. In contrast, the Dutch CAIRO-2 trial [25] has a similar study design to the PACCE trial and involved over 700 patients. Patients received capecitabine, oxaliplatin, and bevacizumab with or without additional cetuximab. As expected, preliminary result reported a significantly higher incidence of grade 3-4 skin rash in the cetuximab-containing arm, without a statistically significant difference in mortality in either arm. The CALGB/SWOG 80405 intergroup trial was a 3-arm study, which randomized patients with metastatic CRC to chemotherapy and cetuximab, chemotherapy and 
TABLE 2: Nonstatistical comparison of the results of the BOND-2 [23] and BOND-1 study [1].

\begin{tabular}{|c|c|c|c|c|}
\hline BOND2 & BOND2 & & BOND1 & BOND1 \\
\hline Cetuximab, irinotecan, and bevacizumab & Cetuximab and bevacizumab & & Cetuximab and irinotecan & Cetuximab alone \\
\hline No. of Patients & 43 & 40 & 218 & 111 \\
\hline Prior treatment with oxaliplatin (\%) & 87 & 89 & 62 & 64 \\
\hline Response rates $(\%)$ & 37 & 20 & 23 & 11 \\
\hline Time to progression (months) & 7.3 & 4.9 & 4.1 & 1.5 \\
\hline Median overall survival (months) & 14.5 & 11.4 & 8.6 & 6.9 \\
\hline
\end{tabular}

bevacizumab, or chemotherapy plus cetuximab and bevacizumab. This study has been suspended following a decision by the CALGB Data and Safety Monitoring Board as from June 2008, pending a protocol revision in view of the data on KRAS mutation (see below) [26]. The reason why the PACCE study failed to demonstrate a survival advantage in the panitumumab-bevacizumab arm remains unclear. It is possible that the dose of the antibodies was inappropriate leading to excessive toxicity and reduced efficacy. Patient selection has also proven critical in the optimal use of antiEGFR antibodies as the use of EGFR staining to predict response and outcome has been severely challenged. Thus, a combination approach of targeted agents in metastatic CRC remains controversial and it is the consensus among gastrointestinal oncologists that such an approach remains experimental.

\section{EGFR Expression in Colorectal Cancer}

EGFR as determined by immunohistochemical (IHC) methods was the first biomarker investigated as a potential predictor of response. It is overexpressed in over $80 \%$ of colorectal cancers [27]. However, EGFR expression as measured by immunohistochemistry does not predict clinical benefit [28, 29]. Initial observations in a small retrospective series by Lenz et al. [30] noted that more than 20\% of EGFR-negative patients developed major objective responses. An extensive retrospective analysis was reported in 2005 by Chung et al. [29]. They reported a response rate of $25 \%$ in EGFR-negative patients (4 out of 16) given cetuximab and irinotecan. This was comparable and indistinguishable from the response rate of $23 \%$ seen in two separate clinical trials with EGFR-positive patients [1, 31]. Given this data, immunohistochemical (IHC) demonstration of EGFR expression is no longer required before starting cetuximab therapy in practice. Similarly, in the phase II trial, the response to treatment with panitumumab in patients with metastatic CRC was similar, irrespective of the level of EGFR protein expression assessed by IHC analysis [32].

\section{Potential Predictors of Response to Anti-EGFR Therapy}

8.1. Activating EGFR Gene Mutations. There has been much interest in determining whether EGFR gene mutations may play a role in affecting response to cetuximab or panitumumab. Previous research in nonsmall cell lung cancer [33] has shown that EGFR TK mutations predict benefit from EGFR TKIs. However, several retrospective studies on tumor biopsies and cell lines found that EGFR gene mutations in CRC are extremely rare $[34,35]$. No significantly different gene mutations were found between responders and nonresponders to treatment.

8.2. EGFR Gene Amplification. It has been hypothesized that DNA is a more stable molecule than a protein and thus EGFR gene copy number maybe more accurate reflection of the EGFR status than IHC expression [36]. In a cohort of patients treated with cetuximab or panitumumab, EGFR gene amplification with increased copy number has been shown to significantly correlate with objective response to treatment [36]. This raises the possibility that patients with high EGFR copy number may be more likely to respond to treatment with anti-EGFR therapies.

8.3. KRAS Gene Mutation. KRAS is a guanosine triphosphate (GTP)-binding protein that acts as a critical on-off switch in cellular growth and survival pathways. It plays a key role in the RAS/MAPK signally pathway located downstream of many growth factor receptors, including EGFR, and involved in carcinogenesis. Mutations of KRAS that result in the constitutive activation of MAPK pathway downstream occurs in about $40 \%$ of colorectal cancers [37]. A retrospective analysis reported by Lièvre et al. [38] analyzed tumor samples from 30 patients treated with cetuximab. A KRAS mutation was found in 13 tumors (43\%) and was significantly associated with absence of response to cetuximab. None of the patients with response to cetuximab harbored a KRAS mutation. The overall survival of patients without KRAS mutation was significantly higher compared with those patients with a mutated tumor $(P=.016$; median survival 16.3 versus 6.9 months). An increase in EGFR copy number was also significantly associated with objective tumor response.

It has been hypothesized that irrespective of the level of EGFR expression, the presence of a KRAS mutation is associated with a constitutive activation of the RAS/MAPK pathway, leading to cell proliferation which cannot be significantly inhibited by cetuximab. KRAS mutations have also been implicated in resistance against EGFR TKIs in lung adenocarcinomas [39].

Large scale retrospective reviews retrieved archived tumor tissue from prior cetuximab and panitumumab trials. KRAS mutation analysis was performed tested in tumor samples collected from over 1000 participants of the "CRYSTAL" 
[8], “OPUS” [40], NCIC-CO.17 trial [41] and panitumumab trials [42]. Beneficial effects of anti-EGFR antibodies were limited to a subgroup of patients with wild-type KRAS tumors. This has led to the recommendation that all patients with advanced CRC who are being considered for cetuximab or panitumumab should undergo KRAS testing, and if the cancer bears a mutated KRAS gene, they should not receive an antibody that targets EGFR.

\section{Conclusion}

Throughout the last decade, significant advancement in our understanding of the molecular mechanisms of metastatic CRC has been made. Anti-EGFR monoclonal antibodies approved for use in the metastatic setting have broadened the therapeutic armamentarium in the treatment of metastatic CRC. The most effective sequence and combinations of antiEGFR therapy with chemotherapy and, or bevacizumab in order to achieve cytotoxic potentiation with limited toxicity need to be addressed. Advances made in the identification of predictive biomarkers such as KRAS mutations allow us to select distinct groups of patients who are most likely to benefit from cetuximab therapy.

\section{References}

[1] H. Hurwitz, L. Fehrenbacher, W. Novotny, et al., "Bevacizumab plus irinotecan, fluorouracil, and leucovorin for metastatic colorectal cancer," The New England Journal of Medicine, vol. 350, no. 23, pp. 2335-2342, 2004.

[2] D. Cunningham, Y. Humblet, S. Siena, et al., "Cetuximab monotherapy and cetuximab plus irinotecan in irinotecanrefractory metastatic colorectal cancer," The New England Journal of Medicine, vol. 351, no. 4, pp. 337-345, 2004.

[3] N. Normanno, A. De Luca, C. Bianco, et al., "Epidermal growth factor receptor (EGFR) signaling in cancer," Gene, vol. 366, no. 1, pp. 2-16, 2006.

[4] J. Mendelsohn and J. Baselga, "Epidermal growth factor receptor targeting in cancer," Seminars in Oncology, vol. 33, no. 4, pp. 369-385, 2006.

[5] N. E. Hynes and H. A. Lane, "ERBB receptors and cancer: the complexity of targeted inhibitors," Nature Reviews Cancer, vol. 5, no. 5, pp. 341-354, 2005.

[6] D. J. Jonker, C. J. O'Callaghan, C. S. Karapetis, et al., "Cetuximab for the treatment of colorectal cancer," The New England Journal of Medicine, vol. 357, no. 20, pp. 2040-2048, 2007.

[7] E. Van Cutsem, I. Lang, G. D’Haens, et al., “KRAS status and efficacy in the first-line treatment of patients with metastatic colorectal cancer (mCRC) treated with FOLFIRI with or without cetuximab: the CRYSTAL experience," Journal of Clinical Oncology, vol. 26, no. 20S, 2008, abstract 2.

[8] C. Bokemeyer, I. Bondarenko, A. Makhson, et al., "Cetuximab plus 5-FU/FA/oxaliplatin (FOLFOX-4) versus FOLFOX4 in the first-line treatment of metastatic colorectal cancer (mCRC): OPUS, a randomized phase II study," Journal of Clinical Oncology, vol. 25, no. 18S, 2007, abstract 4035.

[9] J. Tabernero, E. Van Cutsem, E. Díaz-Rubio, et al., "Phase II trial of cetuximab in combination with fluorouracil, leucovorin, and oxaliplatin in the first-line treatment of metastatic colorectal cancer," Journal of Clinical Oncology, vol. 25, no. 33, pp. 5225-5232, 2007.
[10] E. Van Cutsem, M. Peeters, S. Siena, et al., "Open-label phase III trial of panitumumab plus best supportive care compared with best supportive care alone in patients with chemotherapy-refractory metastatic colorectal cancer," Journal of Clinical Oncology, vol. 25, no. 13, pp. 1658-1664, 2007.

[11] A. E. Wakeling, S. P. Guy, J. R. Woodburn, et al., "ZD1839 (Iressa): an orally active inhibitor of epidermal growth factor signaling with potential for cancer therapy," Cancer Research, vol. 62, no. 20, pp. 5749-5754, 2002.

[12] J. Baselga, D. Rischin, M. Ranson, et al., "Phase I safety, pharmacokinetic, and pharmacodynamic trial of ZD1839, a selective oral epidermal growth factor receptor tyrosine kinase inhibitor, in patients with five selected solid tumor types," Journal of Clinical Oncology, vol. 20, no. 21, pp. 4292-4302, 2002.

[13] M. Ranson, L. A. Hammond, D. Ferry, et al., "ZD1839, a selective oral epidermal growth factor receptor-tyrosine kinase inhibitor, is well tolerated and active in patients with solid, malignant tumors: results of a phase I trial," Journal of Clinical Oncology, vol. 20, no. 9, pp. 2240-2250, 2002.

[14] R. S. Herbst, A.-M. Maddox, M. L. Rothenberg, et al., "Selective oral epidermal growth factor receptor tyrosine kinase inhibitor ZD1839 is generally well-tolerated and has activity in non-small-cell lung cancer and other solid tumors: results of a phase I trial," Journal of Clinical Oncology, vol. 20, no. 18, pp. 3815-3825, 2002.

[15] M. L. Rothenberg, B. LaFleur, D. E. Levy, et al., "Randomized phase II trial of the clinical and biological effects of two dose levels of gefitinib in patients with recurrent colorectal adenocarcinoma," Journal of Clinical Oncology, vol. 23, no. 36, pp. 9265-9274, 2005.

[16] J. T. Hartmann, J. P. Pintoffl, H. Kröning, C. Bokemeyer, M. Holtmann, and T. Höhler, "Gefitinib in combination with oxaliplatin and 5-fluorouracil in irinotecan-refractory patients with colorectal cancer: a phase I study of the arbeits gemeinschaft internistische onkologie (AIO)," Onkologie, vol. 31, no. 5, pp. 237-241, 2008.

[17] T. Kuo, C. D. Cho, J. Halsey, et al., "Phase II study of gefitinib, fluorouracil, leucovorin, and oxaliplatin therapy in previously treated patients with metastatic colorectal cancer," Journal of Clinical Oncology, vol. 23, no. 24, pp. 5613-5619, 2005.

[18] G. A. Fisher, T. Kuo, M. Ramsey, et al., "A phase II study of gefitinib, 5-fluorouracil, leucovorin, and oxaliplatin in previously untreated patients with metastatic colorectal cancer," Clinical Cancer Research, vol. 14, no. 21, pp. 7074-7079, 2008.

[19] J. A. Meyerhardt, K. Stuart, C. S. Fuchs, et al., "Phase II study of FOLFOX, bevacizumab and erlotinib as first-line therapy for patients with metastastic colorectal cancer," Annals of Oncology, vol. 18, no. 7, pp. 1185-1189, 2007.

[20] D. R. Spigel, J. D. Hainsworth, H. Burris, et al., "Phase II study of FOLFOX4, bevacizumab, and erlotinib as first-line therapy in patients with advanced colorectal cancer," in Proceedings of ASCO Gastrointestinal Cancers Annual Symposium, San Francisco, Calif, USA, January 2006, abstract 238.

[21] J. Hecht, E. Mitchell, J. Baranda, et al., "Panitumumab antitumor activity in patients (pts) with metastatic colorectal cancer (mCRC) expressing low (1-9\%) or negative $(<1 \%)$ levels of epidermal growth factor receptor (EGFr)," in Proceedings of ASCO Gastrointestinal Cancer Annual Symposium, San Francisco, Calif, USA, January 2006, abstract 350.

[22] S. Tejpar, M. Peeters, Y. Humblet, et al., "Phase I/II study of cetuximab dose-escalation in patients with metastatic colorectal cancer (mCRC) with no or slight skin reactions on 
cetuximab standard dose treatment (EVEREST): pharmacokinetic (PK), pharmacodynamic (PD) and efficacy data," Journal of Clinical Oncology, vol. 25, no. 18S, 2007, abstract 4037.

[23] L. B. Saltz, H.-J. Lenz, H. L. Kindler, et al., "Randomized phase II trial of cetuximab, bevacizumab, and irinotecan compared with cetuximab and bevacizumab alone in irinotecanrefractory colorectal cancer: the BOND-2 study," Journal of Clinical Oncology, vol. 25, no. 29, pp. 4557-4561, 2007.

[24] J. Hecht, T. Chidiac, E. Mitchell, et al., "An interim analysis of efficacy and safety from a randomized controlled trial of panitumumab with chemotherapy pluse bevacizumab in metastastic colorectal cancer," in Proceedings of the 9th World Congress on Gastrointestinal Cancer, Barcelona, Spain, June 2007, abstract 33 .

[25] J. Tol, M. Koopman, C. J. Rodenburg, et al., "A randomised phase III study on capecitabine, oxaliplatin and bevacizumab with or without cetuximab in first-line advanced colorectal cancer, the CAIRO2 study of the Dutch Colorectal Cancer Group (DCCG). An interim analysis of toxicity," Annals of Oncology, vol. 19, no. 4, pp. 734-738, 2008.

[26] A. P. Venook, C. D. Blanke, D. Niedzwiecki, et al., "Revisiting the cancer and leukemia group B/southwest oncology group 80405 trial: a phase III trial of chemotherapy and biologic agents for patients with untreated advanced colorectal adenocarcinoma," Clinical Colorectal Cancer, vol. 6, no. 7, pp. 536538, 2007.

[27] J.-P. Spano, C. Lagorce, D. Atlan, et al., "Impact of EGFR expression on colorectal cancer patient prognosis and survival," Annals of Oncology, vol. 16, no. 1, pp. 102-108, 2005.

[28] R. Adams and T. Maughan, "Predicting response to epidermal growth factor receptor-targeted therapy in colorectal cancer," Expert Review of Anticancer Therapy, vol. 7, no. 4, pp. 503-518, 2007.

[29] K. Y. Chung, J. Shia, N. E. Kemeny, et al., "Cetuximab shows activity in colorectal cancer patients with tumors that do not express the epidermal growth factor receptor by immunohistochemistry," Journal of Clinical Oncology, vol. 23, no. 9, pp. 1803-1810, 2005.

[30] H.-J. Lenz, E. Van Cutsem, S. Khambata-Ford, et al., "Multicenter phase II and translational study of cetuximab in metastatic colorectal carcinoma refractory to irinotecan, oxaliplatin, and fluoropyrimidines," Journal of Clinical Oncology, vol. 24, no. 30, pp. 4914-4921, 2006.

[31] L. B. Saltz, N. J. Meropol, P. J. Loehrer Sr., M. N. Needle, J. Kopit, and R. J. Mayer, "Phase II trial of cetuximab in patients with refractory colorectal cancer that expresses the epidermal growth factor receptor," Journal of Clinical Oncology, vol. 22, no. 7, pp. 1201-1208, 2004.

[32] D. J. Freeman, T. Juan, M. Reiner, et al., "Association of Kras mutational status and clinical outcomes in patients with metastatic colorectal cancer receiving panitumumab alone," Clinical Colorectal Cancer, vol. 7, no. 3, pp. 184-190, 2008.

[33] T. J. Lynch, D. W. Bell, R. Sordella, et al., "Activating mutations in the epidermal growth factor receptor underlying responsiveness of non-small-cell lung cancer to gefitinib," The New England Journal of Medicine, vol. 350, no. 21, pp. 21292139, 2004.

[34] T. D. Barber, B. Vogelstein, K. W. Kinzler, and V. E. Velculescu, "Somatic mutations of EGFR in colorectal cancers and glioblastomas," The New England Journal of Medicine, vol. 351, no. 27, pp. 2883-2885, 2004.

[35] H. Nagahara, K. Mimori, M. Ohta, et al., "Somatic mutations of epidermal growth factor receptor in colorectal carcinoma," Clinical Cancer Research, vol. 11, no. 4, pp. 1368-1371, 2005.
[36] M. Moroni, S. Veronese, S. Benvenuti, et al., "Gene copy number for epidermal growth factor receptor (EGFR) and clinical response to antiEGFR treatment in colorectal cancer: a cohort study," The Lancet Oncology, vol. 6, no. 5, pp. 279-286, 2005.

[37] H. J. N. Andreyev, A. R. Norman, D. Cunningham, J. R. Oates, and P. A. Clarke, "Kirsten ras mutations in patients with colorectal cancer: the multicenter "RASCAL" study," Journal of the National Cancer Institute, vol. 90, no. 9, pp. 675-684, 1998.

[38] A. Lièvre, J.-B. Bachet, D. Le Corre, et al., "KRAS mutation status is predictive of response to cetuximab therapy in colorectal cancer," Cancer Research, vol. 66, no. 8, pp. 39923995, 2006.

[39] W. Pao, T. Y. Wang, G. J. Riely, et al., “KRAS mutations and primary resistance of lung adenocarcinomas to gefitinib or erlotinib," PLoS Medicine, vol. 2, no. 1, p. e17, 2005.

[40] C. Bokemeyer, I. Bondarenko, J. Hartmann, et al., "KRAS status and efficacy of first-line treatment of patients with metastatic colorectal cancer (mCRC) with FOLFOX with or without cetuximab: the OPUS experience," Journal of Clinical Oncology, vol. 26, no. 15S, 2008, abstract 4000.

[41] C. S. Karapetis, S. Khambata-Ford, D. J. Jonker, et al., "K-ras mutations and benefit from cetuximab in advanced colorectal cancer," The New England Journal of Medicine, vol. 359, no. 17, pp. 1757-1765, 2008.

[42] R. G. Amado, M. Wolf, M. Peeters, et al., "Wild-type KRAS is required for panitumumab efficacy in patients with metastatic colorectal cancer," Journal of Clinical Oncology, vol. 26, no. 10, pp. 1626-1634, 2008. 


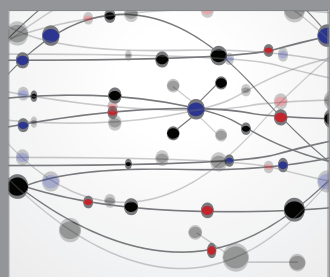

The Scientific World Journal
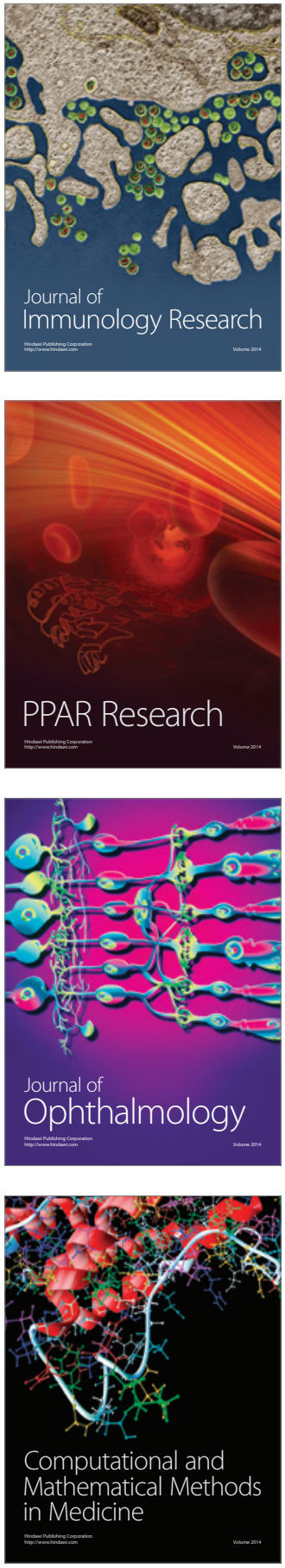

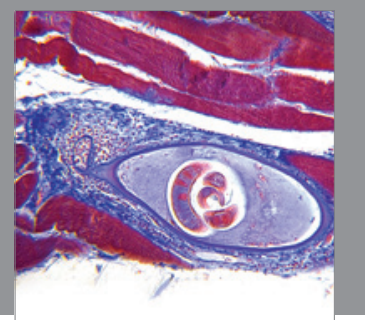

Gastroenterology

Research and Practice
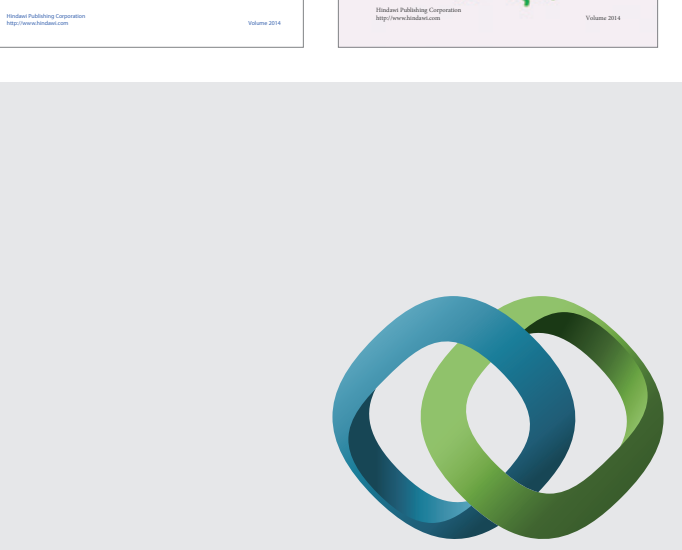

\section{Hindawi}

Submit your manuscripts at

http://www.hindawi.com
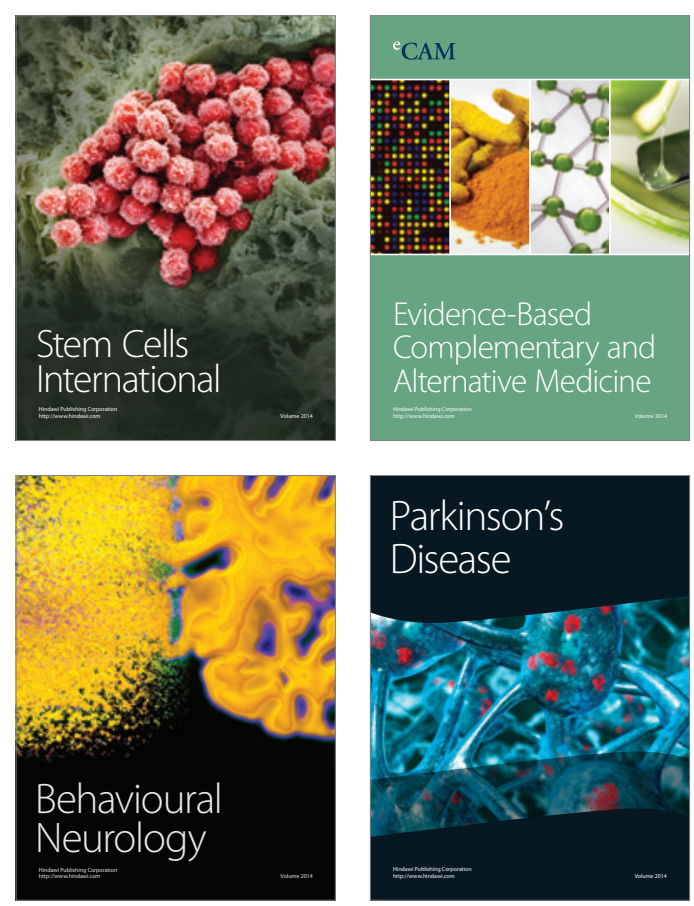

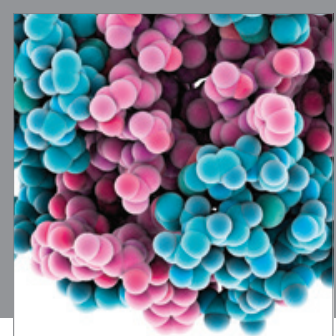

Journal of
Diabetes Research

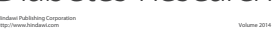

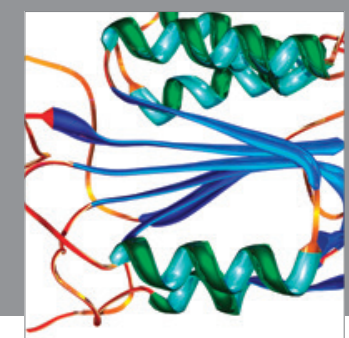

Disease Markers
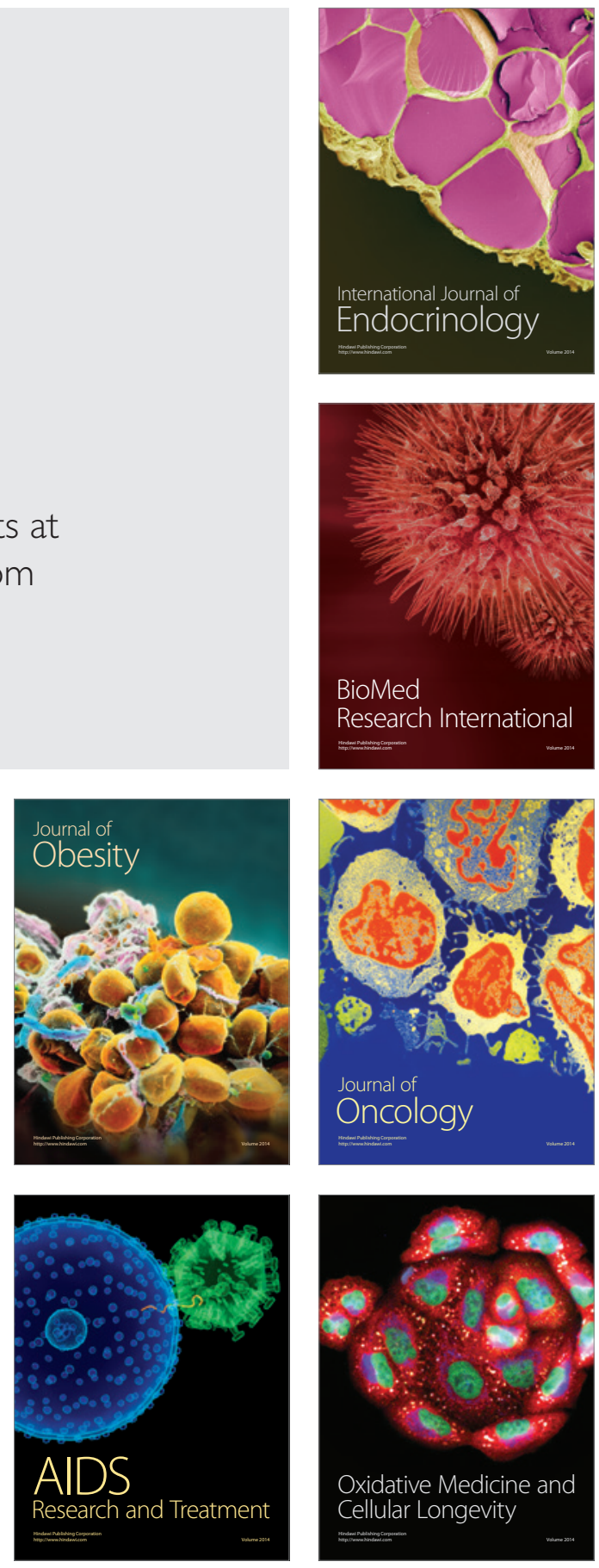